Original article

\title{
Analysis of dental esthetic proportions in a Spanish population sample
}

\author{
Samuel Rodríguez-López ${ }^{1}$, Matías Ferrán Escobedo Martínez ${ }^{1)}$, Jorge Pesquera Velasco²), Luis Junquera ${ }^{3)}$, \\ and María García-Pola ${ }^{3)}$
}

Department of Operative Dentistry, University School of Dental Medicine, University of Oviedo, Oviedo, Spain

2) Institute of Biomedicine, University of León, León, Spain

${ }^{3}$ Department of Oral and Maxillofacial Surgery and Oral Medicine, University School of Dental Medicine, University of Oviedo, Oviedo, Spain

\begin{abstract}
Purpose: The present study aimed to analyze in a Spanish population sample the compliance of the anterior maxillary teeth to the dental esthetic proportions described in the literature.

Methods: Photographs of the smiles of 78 individuals were calibrated and digitally analyzed considering the following proportions: golden proportion (GP), recurring esthetic dental (RED), golden percentage (GPG), Preston's proportion and Modified golden percentage (MGPG). For statistical analysis, the $t$-test of an independent sample was applied, and compliance percentages for each standard were recorded.

Results: The existence of RED $70 \%$ or $80 \%$ has not been registered. The percentages of compliance with GP were within a range between $0 \%$ and $16 \%$. The mean tooth width ratios were adjusted to the values described by Preston $(P>0.05)$, but the compliance percentages were low $(3.33-25 \%)$. GPG only presented high percentages of compliance in the lateral incisors (53.33-62.5\%). MGPG showed the highest percentages of compliance (50$68 \%)$.

Conclusion: After analyzing a Spanish population sample using smile photographs for the first time, GP, RED, GPG and Preston's proportion standards are not fulfilled and therefore, not suitable for treatments that seek a smile that reproduces natural principles. However, the values designated by MGPG with a deviation of $\pm 1 \%$ are largely applicable for treatments that aim for a natural smile.
\end{abstract}

Keywords; golden percentage, golden proportion, modified golden percentage, recurring esthetic dental, smile aesthetics

\section{Introduction}

Harmony and symmetry in a smile are considered essential when programming dental treatments that involve the maxillary anterior teeth. A frontal view appreciation of the teeth width has given rise to multiple studies that have attempted to develop equation-based guidelines that ensure aesthetic results.

Lombardi, in 1973, based on the descriptions and balance of classical Greek art, established proportional measurements between premolar teeth to consider an esthetic denture [1]. Later Levin [2] defined the golden proportion (GP), as one in which, starting from the midline, the width of each successive tooth decreases in the same proportion, progressing distally. Thus, the width of the lateral incisor (LA) should represent $62 \%$ of the central one (CE) and the width of the canine (CA) $62 \%$ of the lateral incisor (Fig. 1A) [2]. In 1993, Preston [3], from 58 dental casts of American students, recorded GP compliance in $17 \%$ of the sample, and proposed new ratios of $66 \%$ between $\mathrm{LA} / \mathrm{CE}$ and $84 \%$ between $\mathrm{CA} / \mathrm{LA}$ (Fig. 1B).

Snow described the golden percentage guide (GPG) in 1999 [4] to correct some difficulties that arose when applying GP, and evaluated the perceived width of each tooth within the intercanine space (ICW). In this

Correspondence to Dr. Samuel Rodríguez-López, Department of Surgery and Medical-Surgical Specialties, Oviedo University, Julián Clavería, Oviedo 33006, Spain

E-mail: samuelopezgij@gmail.com

J-STAGE Advance Publication: May 19, 2021

Color figures can be viewed in the online issue at J-STAGE.

doi.org/10.2334/josnusd.21-0019

DN/JST.JSTAGE/josnusd/21-0019 way, the central incisors would each represent $25 \%$ of the space, each lateral $15 \%$ and each canine, $10 \%$ (Fig. 1C)

Ward, in 2001, described recurring esthetic dental (RED) [5]. Like $\mathrm{GP}$, it calculates the width ratio between LA/CE and CA/LA expressed in percentages, but RED requirements are met when the result of these two operations is numerically equal and are in a range between $62 \%$ and $80 \%$, the RED proportion being $62 \%$ equivalent to the GP (Fig. 1D). Unlike the previous ones, this standard is flexible, and can be applied to different tooth widths based on clinical criteria such as facial morphology or size [6,7].

Some studies which have analyzed these esthetic proportions provide heterogeneous results. Different investigations have shown that samples from dental students from India [8], China [9], Turkey [10], Iran [11] or Hungary [12] do not meet the GP standard. In other studies, GP is met in a small percentage of the sample, e.g., it was observed in $7.1 \%$ of a Brazilian population sample [13]. In two studies from India, the principles were met between $11.4 \%$ [14] and 25\% [15], in Nepalese 14\% [16] and in a population of Arabic origin around 30\% [17]. RED compliance has been recorded at $4 \%$ and $6.6 \%$ in two samples from East India $[18,19]$. Other studies looked at compliance individually in a single tooth $[15,20,21]$. However, studies carried out in the United Kingdom (UK) [22] and India [23] did not record compliance with the RED standard.

Several studies that focused on GPG values recorded similar and constant mean tooth width values. They deviated between $5 \%$ and $0.5 \%$ from the values described in the standard, and considered this proportion as the most appropriate $[15,16]$. Several researchers have proposed that GPG can be applied by adopting certain corrections $[17,19]$. Recently, Kalia et al. [22] proposed the modified golden percentage (MGPG), in which the CE should account for $22.5 \%$, the LA $15 \%$ and the CA $12.5 \%$ of the ICW (Fig. 1C) [22]. However, Calçada et al. [24] consider that the most appropriate proportion is Preston>s proportion.

The aim of this study was to analyze, in a Spanish sample, the width of the maxillary anterior teeth in a frontal view and the compliance of the dental esthetic proportions described in GP, Preston's proportion, RED $70 \%$ and $80 \%$, GPG and MGPG. This was done by comparing the mean width ratios of the maxillary anterior teeth and by the compliance percentages of the sample with the values that mark each esthetic proportion, taking into account a deviation of $\pm 1 \%$. The hypothesis was that the general population presents a trend of greater compliance with a dental esthetic proportion, following a mathematical rule.

\section{Materials and Methods}

\section{Study sample}

The University of Oviedo review board decided that this study did not need approval as it did not involve patients or any type of therapeutic treatment. Data were gathered by taking standardized photographs of members and students of the Faculty of Medicine and Health Sciences of the University of Oviedo (Spain) and of the Master of Surgery and Implantoprosthesis of the University of León (Spain). Photographs were taken between February 15, 2020 and March 5, 2020. The sample population comprised 78 individuals, 48 women and 30 men. Mean ages were 28.37 years for the whole population (range: $20-64$ years), 29.00 years for women and 27.27 for men.

Participants had to meet the following inclusion criteria: 1) >18 years; 2) no absence of the maxillary anterior teeth; 3) absence of diastemas or bad positions of the maxillary anterior teeth; 4) no loss of tooth structure; 5) no history of restorative and/or prosthetic treatments, 6) no history of 

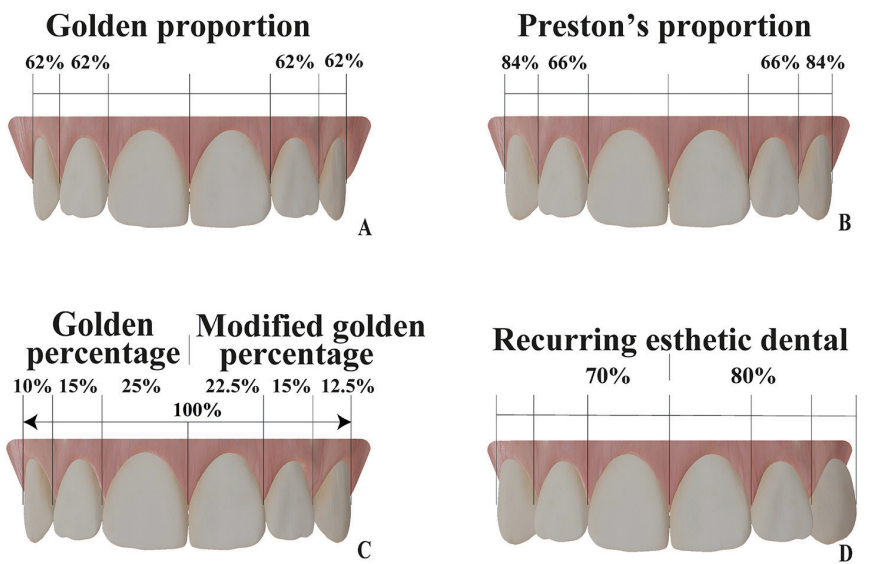

Fig. 1 Graphics representing the different esthetic dental proportions
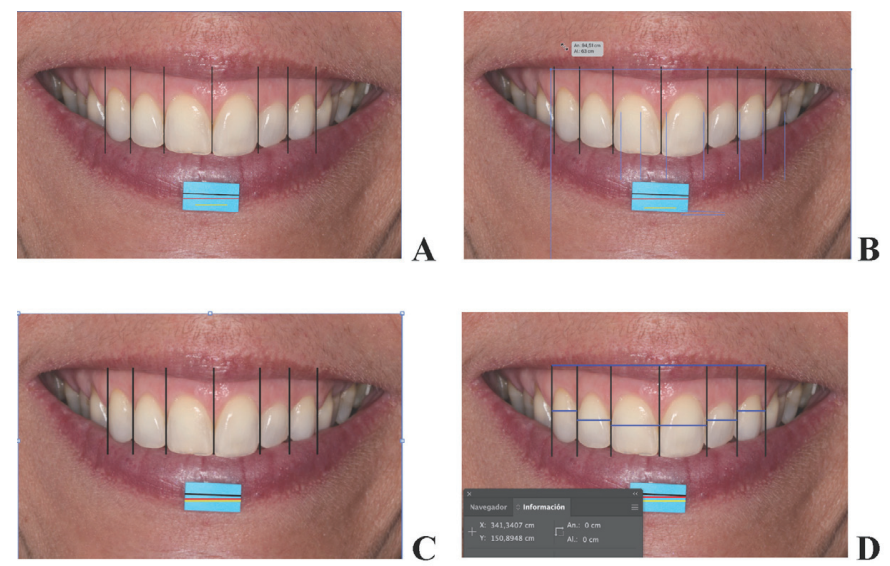

Fig. 2 Images of the analysis protocol and photo editing. A: capture of Adobe Illustrator. Smile with vertical lines drawn. The black and red horizontal lines are plotted on the adherent tab for scale, and the yellow one measures $1 \mathrm{~cm}$. B: capture of Adobe Illustrator. Image illustrating photo dimension scaling with vertical lines and black and red horizontal lines selected. The proportional reduction tool of the software program was used. C: capture of Adobe Illustrator. Image illustrating that both lines are almost entirely aligned. D: capture of Adobe Illustrator. Image with all measurement lines drawn on the image already calibrated and the measurement tool panel open.

periodontal disease; 7) no history of surgical treatments in the maxillary anterior region, and 8) absence of orthodontic appliances when the photographs were taken.

The exclusion criteria were as follows: 1) participants with crowding, diastemata or rotation of teeth in the anterior-superior region; 2) evidence of gingival alterations, and 3) irregularities in the upper anterior teeth (loss of tooth structure due to wear affecting the mesiodistal width, fracture, caries or dental treatments).

\section{Photo recording protocol}

The images were taken following the guidelines of the "American Academy of Cosmetic Dentistry" (AACD), following the modality "full smile frontal view, 1:2 (1:3) magnification and non-retracted view" (https://aacd. com. Accessed March 27, 2021). In addition to the AACD criteria and to calibrate the images, an adhesive with standardized dimensions was placed on the lower lip of each participant, as a tab for scale.

The photographs were taken by an operator (S-R-L), in two rooms with participants in identical lightening. The subjects' heads were positioned with Frankfort plane parallel to the ground by using an orthopantomography device (Proline XC; Planmeca, Helsinki, Finland). An APS-C reflex camera (Canon EOS 80D; Canon Inc., Tokyo, Japan) equipped with a macro lens (Canon EF $100 \mathrm{~mm} \mathrm{f/2.8} \mathrm{Macro} \mathrm{USM;} \mathrm{Canon} \mathrm{Inc.)} \mathrm{and} \mathrm{a} \mathrm{ring}$ flash (Macro ring lite MR-14EXII; Canon Inc.) was used. The parameters used were: ISO: 200; shutter speed: 1/125; diaphragm aperture f: 20; and distance to the target: $0.49 \mathrm{~m}$. Since the digital camera had an APS-C sensor $(22.2 \mathrm{~mm} \times 14.8 \mathrm{~mm})$, a magnification ratio of 1:3 was applied to achieve the 1:2 magnification defined in the protocol of the AACD.

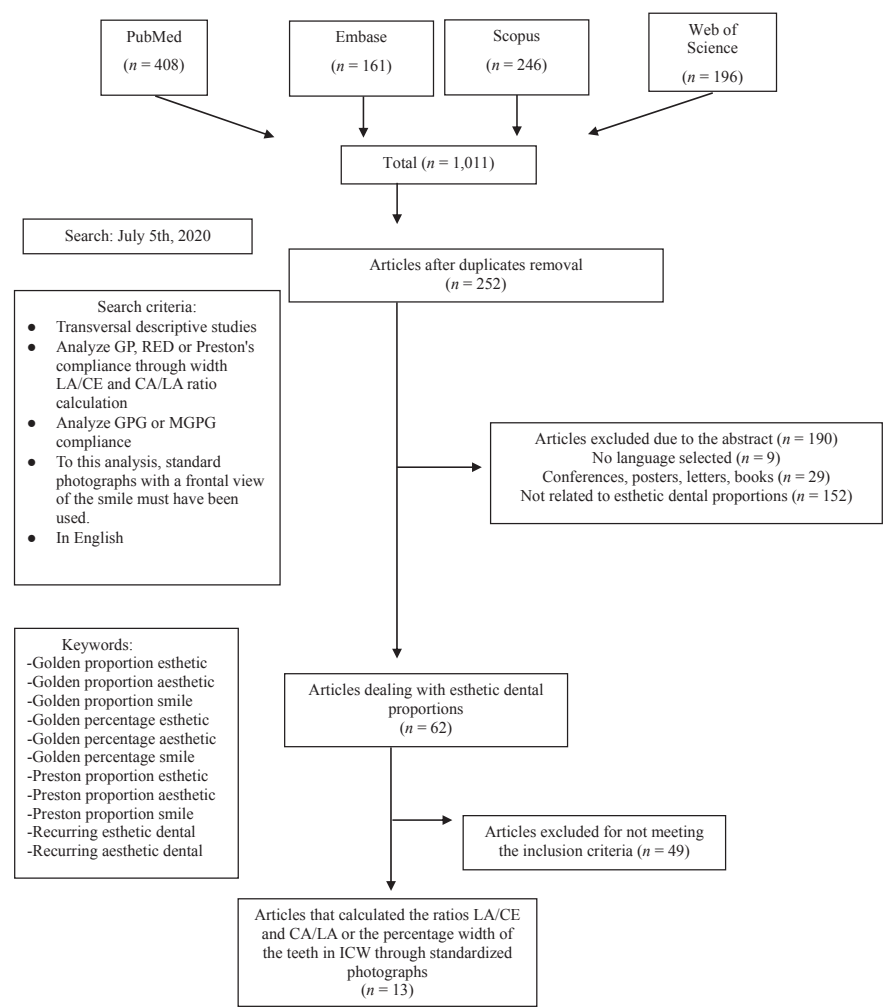

Fig. 3 Flow diagram of data collection process. GP, golden proportion, RED, recurring esthetic dental; GPG, golden percentage; ICW, intercanine width

\section{Protocol for analysis of photographs}

Analysis of photographs was performed using a vector graphics editor software (Adobe Illustrator 23.1.1; Adobe Systems Inc., San Jose, CA, USA). Images were calibrated to obtain more precise measurements of the exposed tooth portion in the frontal view of the smile. For this, vinyl adhesives standardized at $1 \mathrm{~cm}$ (Macal 8300 Pro; MACtac Americas LLC, Stow, OH, USA) cut by a Cutter Plotter (Cutting pro FC7000-160; Graphtec Corporation; Tokyo, Japan) were made.

In Adobe Illustrator, the central points of proximal contacts of the maxillary anterior teeth were marked and, over these, vertical lines parallel to the Y-axis were drawn. Later, a line as long as the tab for scale, located on the inferior lip, was drawn (black line) (Fig. 2A). This line was duplicated, and its orientation matched the $\mathrm{X}$-axis (red line) (Fig. 2A). Then another line, parallel to the red line, was drawn, instructing the program to give it a length of $1 \mathrm{~cm}$ (yellow line). Calibration ended when the size of the image was proportionally reduced with the vertical, black and red lines linked, until the red line coincides in dimensions with the yellow line of $1 \mathrm{~cm}$, generating a 1:1 scale (Fig. 2B, C). Once the image was calibrated, the dental dimensions were calculated with the software measurement tool (Fig. 2D).

\section{Statistical analysis}

The variables analyzed in the sample studied were gender, age, width of each tooth (CE, LA, CA; in $\mathrm{mm}$ ), the intercanine space (ICW; in $\mathrm{mm}$ ), the percentage width of each tooth in the ICW and the percentage ratios between LA/CE and CA/LA teeth. The Student $t$-test was used for comparison in the case of quantitative variables after checking the normality hypothesis through the Shapiro-Wilks test. The differences observed in the dimensions of the teeth of the sample in relation to the proportions studied were evaluated. Differences based on sex were also evaluated. The reference for GP was $62 \%$ for Preston's Proportion 66\% (LA/CE) and $84 \%$ (CA / CE); for GPG the proportion 25\% (CE), $15 \%$ (LA) and $10 \%$ (CA); for MGPG the proportion was $22.5 \%$ (CE), $15 \%$ (LA) and $12.5 \%$ (CA); and for RED, $70 \%$ and $80 \%$. A $95 \%$ confidence interval of the mean value for each proportion studied was considered, with a significance level of 0.05 . Also, the percentage of compliance with the mathematical norms was analyzed with a range of $\pm 1 \%$. The statistical analysis was carried out with the software R 3.4.4.2018 (R Core Team. R Foundation for Statistical Computing, Vienna, Austria). 
Table 1 Tooth width and intercanine width in $\mathrm{mm}$

\begin{tabular}{|c|c|c|c|c|}
\hline Tooth & Male (SD) & Female (SD) & $P$-value & All participants (SD) \\
\hline CA right & $5.07(0.49)$ & $4.91(0.48)$ & 0.156 & $4.97(0.49)$ \\
\hline LA right & $5.59(0.61)$ & $5.76(0.52)$ & 0.785 & $5.77(0.56)$ \\
\hline CE right & $8.71(0.55)$ & $8.44(0.78)$ & 0.071 & $8.54(0.71)$ \\
\hline CE left & $8.55(0.60)$ & $8.19(0.75)$ & 0.028 & $8.33(0.72)$ \\
\hline LA left & $5.55(0.59)$ & $5.38(0.55)$ & 0.213 & $5.44(0.57)$ \\
\hline CA left & $4.76(0.62)$ & $4.50(0.55)$ & 0.061 & $4.60(0.59)$ \\
\hline ICW & $38.51(1.83)$ & $37.42(2.16)$ & 0.005 & $37.67(2.13)$ \\
\hline
\end{tabular}

SD, standard deviation; $\mathrm{CA}$, canine; LA, lateral incisor; $\mathrm{CE}$, central incisor; ICW, intercanine width

Table 2 Comparison between tooth width ratios and reference proportions

\begin{tabular}{|c|c|c|c|c|c|}
\hline Ratio & Mean values observed \% (C.I) & $\begin{array}{c}\text { Golden proportion }(62 \%) \\
(P \text {-value })\end{array}$ & $\begin{array}{c}\text { Preston (66\% and } 84 \%) \\
\text { ( } P \text {-value) }\end{array}$ & $\begin{array}{l}\text { RED }(70 \%) \\
(P \text {-value })\end{array}$ & $\begin{array}{l}\text { RED }(80 \%) \\
(P \text {-value })\end{array}$ \\
\hline \multicolumn{6}{|l|}{ All participants } \\
\hline Right CA/LA & $86.9(84.3-89.4)$ & $<0.001$ & 0.029 & $<0.001$ & $<0.001$ \\
\hline Right LA/CE & $67.8(66.4-69.2)$ & $<0.001$ & 0.014 & 0.002 & $<0.001$ \\
\hline Left LA/CE & $65.7(64.0-67.3)$ & $<0.001$ & 0.690 & $<0.001$ & $<0.001$ \\
\hline Left CA/LA & $85.3(82.4-88.1)$ & $<0.001$ & 0.389 & $<0.001$ & 0.001 \\
\hline \multicolumn{6}{|l|}{ Female } \\
\hline Right CA/LA & $85.9(82.8-89.0)$ & $<0.001$ & 0.229 & $<0.001$ & $<0.001$ \\
\hline Right LA/CE & $68.5(66.8-70.2)$ & $<0.001$ & 0.003 & 0.080 & $<0.001$ \\
\hline Left LA/CE & $66.1(63.7-68.5)$ & 0.001 & 0.912 & 0.002 & $<0.001$ \\
\hline Left CA/LA & $84.6(80.7-88.5)$ & $<0.001$ & 0.767 & $<0.001$ & 0.022 \\
\hline \multicolumn{6}{|l|}{ Male } \\
\hline Right CA/LA & $88.5(83.7-93.2)$ & $<0.001$ & 0.063 & $<0.001$ & 0.001 \\
\hline Right LA/CE & $66.6(64.1-69.1)$ & 0.001 & 0.652 & 0.009 & $<0.001$ \\
\hline Left CA/LA & $86.3(81.9-90.8)$ & $<0.001$ & 0.291 & $<0.001$ & 0.007 \\
\hline
\end{tabular}

RED, recurring esthetic dental; C.I, confidence interval; CA, canine; LA, lateral incisor; CE, central incisor

Table 3 Percentages of compliance with golden proportion, RED 70\% and 80\% and Preston's proportion

\begin{tabular}{|c|c|c|c|c|c|c|c|c|c|c|c|c|}
\hline \multirow[t]{2}{*}{ Ratio } & \multicolumn{3}{|c|}{$\begin{array}{l}\text { Golden proportion } \\
(62 \% \pm 1 \%)\end{array}$} & \multicolumn{3}{|c|}{$\begin{array}{c}\text { Preston's proportion } \\
(64 \% \pm 1 \% \text { and } 84 \% \pm 1 \%)\end{array}$} & \multicolumn{3}{|c|}{$\operatorname{RED}(70 \% \pm 1 \%)$} & \multicolumn{3}{|c|}{$\operatorname{RED}(80 \% \pm 1 \%)$} \\
\hline & All & Female & Male & All & Female & Male & All & Female & Male & All & Female & Male \\
\hline Right CA/LA & $1.3 \%$ & $2.1 \%$ & $0.0 \%$ & $10.3 \%$ & $10.4 \%$ & $10.0 \%$ & $1.3 \%$ & $2.1 \%$ & $0.00 \%$ & $12.8 \%$ & $12.5 \%$ & $13.3 \%$ \\
\hline Right LA/CE & $3.9 \%$ & $4.2 \%$ & $3.3 \%$ & $19.2 \%$ & $25.0 \%$ & $10.0 \%$ & $14.1 \%$ & $12.5 \%$ & $16.7 \%$ & $0.0 \%$ & $0.0 \%$ & $0.0 \%$ \\
\hline Left LA/CE & $14.1 \%$ & $12.5 \%$ & $16.7 \%$ & $9.0 \%$ & $10.4 \%$ & $6.7 \%$ & $11.5 \%$ & $14.6 \%$ & $6.7 \%$ & $0.0 \%$ & $0.0 \%$ & $0.0 \%$ \\
\hline Left CA/LA & $2.6 \%$ & $2.1 \%$ & $3.3 \%$ & $2.6 \%$ & $2.1 \%$ & $3.3 \%$ & $3.9 \%$ & $2.1 \%$ & $6.7 \%$ & $7.7 \%$ & $6.3 \%$ & $10.0 \%$ \\
\hline
\end{tabular}

RED, recurring esthetic dental; CA, canine; LA, lateral incisor; $\mathrm{CE}$, central incisor

\section{Bibliographic review}

To compare the results of this work with the bibliographic information on the subject, a literature review was carried out. A summary of the methodology used is reflected in Fig. 3.

\section{Results}

Table 1 shows the mean values, in $\mathrm{mm}$, of the mesiodistal widths of the maxillary anterior teeth in a frontal view of men and women. The mean width of the teeth of the men was greater in all the comparisons. However, statistically significant differences only were observed when considering the width of the left CE $(P=0.028)$ and the ICW $(P=0.005)$.

\section{Golden proportion compliance}

Table 2 shows the mean values (\%) of the LA/CE and CA/LA ratios of the analyzed sample. When comparing these measures with the percentage determined by the norm (62\%), significant differences were found in all cases; therefore, the values are not in the GP. Table 3 shows the percentages of compliance with GP, with a deviation of $\pm 1 \%$. In the comparison, the lowest values $(0.00-3.33 \%)$ were recorded for CA/LA, whereas the highest values did not reach 17\%, and were between 3.33 and $16.67 \%$ in $\mathrm{LA} / \mathrm{CE}$.

\section{Preston's proportion compliance}

Table 2 shows the mean values resulting from the calculation of the $\mathrm{LA} / \mathrm{CE}$ and $\mathrm{CA} / \mathrm{LA}$ ratios. When considering the total sample, their average values were adjusted to Preston's Proportion in the ratios CA/LA and LA/CE on the left side $(P=0.690$ and $P=0.389)$. Women showed compliance in all ratios except in LA/CE right $(P=0.003)$. Men's mean values were adjusted in all comparisons $(P>0.05)$. Considering the percentages of compliance with Preston's proportion (Table 3), the whole sample had higher values recorded on the right side $19 \%(\mathrm{LA} / \mathrm{CE})$ and $10.26 \%(\mathrm{CA} / \mathrm{CE})$ than on the left side $8.97 \%(\mathrm{LA} / \mathrm{CE})$ and $2.56 \%(\mathrm{CA} / \mathrm{CE})$. Women presented the highest and lowest percentage of compliance of all the comparative ones; $25 \%$ in the ratio LA/CE right and $2.08 \%$ between CA/LA left. Men showed compliance percentages of $10 \%$ in the ratios on the right side and in those on the left side, $6.67 \%$ between the left LA/CE and $3.33 \%$ between the CA/LA.

\section{RED compliance $(\mathbf{7 0 \%}$ and $80 \%)$}

Table 2 compares the mean values of the LA/CE and CA/LA ratios on both sides with the RED ratio of $70 \%$ and $80 \%$. The average data in accordance with RED proportion were only recorded in the mean value of the LA/CE ratio on the right side of women with the RED $70 \%$ ratio $(P=0.080)$. Likewise, the resulting values of the LA/CE and CA/LA ratios on each side are not numerically equal in any of the cases analyzed, so the sample did not conform to RED proportion. When analyzing the percentages of compliance with RED $70 \%$ of the total sample, including men and women, they were higher in the ratios between LA/CE (11.54-16.67\%) than between CA/LA $(0.00 \%$ to $6.67 \%)$. In RED $80 \%$, considering all of the sample, the percentages were $0.00 \%$ in the ratios between $\mathrm{LA} / \mathrm{CE}$ and from $6.25 \%$ to $13.33 \%$ between $\mathrm{CA} / \mathrm{LA}$. 
Table 4 Tooth widths in the ICW space vs. reference proportions

\begin{tabular}{|c|c|c|c|}
\hline Tooth & Mean values observed \% (C.I) & Golden percentage ( $P$-value) & Modified golden percentage ( $P$-value) \\
\hline \multicolumn{4}{|l|}{ All participants } \\
\hline Right CA & $13.2(12.9-13.5)$ & $<0.001$ & $<0.001$ \\
\hline Right LA & $15.3(15.0-15.6)$ & 0.023 & 0.024 \\
\hline Right CE & $22.7(22.3-23.0)$ & $<0.001$ & 0.295 \\
\hline Left CE & $22.1(21.8-22.4)$ & $<0.001$ & 0.013 \\
\hline Left LA & $14.4(14.2-14-7)$ & $<0.001$ & $<0.001$ \\
\hline Left CA & $12.2(11.9-12.6)$ & $<0.001$ & 0.094 \\
\hline \multicolumn{4}{|l|}{ Female } \\
\hline Right CA & $13.2(12.7-13.7)$ & $<0.001$ & 0.014 \\
\hline Right LA & $15.0(14.5-15.5)$ & 0.913 & 0.091 \\
\hline Right CE & $22.6(22-3-23.0)$ & $<0.001$ & 0.505 \\
\hline Left CE & $22.2(21.8-22.6)$ & $<0.001$ & 0.094 \\
\hline Left LA & $14.4(14.0-14.8)$ & 0.007 & 0.007 \\
\hline Left CA & $12.4(11.8-12.9)$ & $<0.001$ & 0.620 \\
\hline \multicolumn{4}{|l|}{ Male } \\
\hline Right CA & $13.2(12.9-13.6)$ & $<0.001$ & $<0.001$ \\
\hline Right LA & $15.5(15.2-15.8)$ & 0.003 & 0.003 \\
\hline Right CE & $22.7(22.2-23.2)$ & $<0.001$ & 0.401 \\
\hline Left CE & $22.1(21.6-22.5)$ & $<0.001$ & 0.054 \\
\hline Left LA & $14.5(14.1-14.8)$ & 0.004 & 0.004 \\
\hline Left CA & $12.1(11.7-12.5)$ & $<0.001$ & 0.078 \\
\hline
\end{tabular}

ICW, intercanine width; C.I, confidence interval; CA, canine; LA, lateral incisor; $\mathrm{CE}$, central incisor

Table 5 Percentages of compliance with the golden percentage and the modified golden percentage

\begin{tabular}{|c|c|c|c|c|c|c|}
\hline \multirow[t]{2}{*}{ Tooth } & \multicolumn{3}{|c|}{ Golden percentage $( \pm 1 \%)$} & \multicolumn{3}{|c|}{ Modified golden percentage $( \pm 1 \%)$} \\
\hline & All & Female & Male & All & Female & Male \\
\hline Right CA & $2.6 \%$ & $2.1 \%$ & $23.3 \%$ & $50.0 \%$ & $50.0 \%$ & $50.0 \%$ \\
\hline Right LA & $55.1 \%$ & $56.3 \%$ & $53.3 \%$ & $55.1 \%$ & $56.3 \%$ & $53.3 \%$ \\
\hline Right CE & $10.3 \%$ & $12.5 \%$ & $6.7 \%$ & $68.0 \%$ & $68.8 \%$ & $66.7 \%$ \\
\hline Left CE & $5.1 \%$ & $6.3 \%$ & $3.3 \%$ & $66.7 \%$ & $66.7 \%$ & $66.7 \%$ \\
\hline Left LA & $60.3 \%$ & $62.5 \%$ & $56.7 \%$ & $60.3 \%$ & $62.5 \%$ & $56.7 \%$ \\
\hline Left CA & $23.1 \%$ & $29.2 \%$ & $13.3 \%$ & $53.9 \%$ & $52.1 \%$ & $56.7 \%$ \\
\hline
\end{tabular}

$\mathrm{CA}$, canine; LA, lateral incisor; $\mathrm{CE}$, central incisor

\section{Golden percentage compliance}

Table 4 shows the mean width percentages occupied by the upper anterior teeth in the ICW. The average right LA in women were the only conforming GPG $(15.03 \%, P=0.913)$. Table 5 shows the percentages of compliance with GPG with a deviation of $\pm 1 \%$. The lowest percentages recorded were in the CE (3.33-12.50\%), followed by the CA $(2.08-29.17 \%)$, and only reached values higher than $50 \%$ in the LA $(53.33-62.50 \%)$.

\section{Modified golden percentage compliance}

Comparisons between the mean widths of the maxillary anterior teeth recorded against the reference values of MGPG are shown in Table 4. In the analysis of the total sample, the right $\mathrm{CE}$ and the left $\mathrm{AC}$ fit the norm ( $P=0.295$ and $P=0.094)$. Comparing women's data, the mean of the right LA $(P=0.091)$, the right and left CE $(P=0.505$ and $P=0.094)$ and the left AC $(P=0.620)$ conformed to MGPG. In the case of men, all the mean values of the $\mathrm{CE}(P=0.401$ and $P=0.054)$ and the left $\mathrm{CA}(P=0.078)$ were adjusted to MGPG. Table 5 shows the percentages of compliance with the MGPG with a deviation of $\pm 1 \%$. The compliance percentages in the CA were distributed in a range between $50.00 \%$ and $56.67 \%$, LA between $53.33 \%$ and $62.50 \%$ and CE between $66.67 \%$ and $68.75 \%$.

\section{Bibliographic review results}

The results of previous works that studied GP, RED and Preston's proportion are reflected in Table $6[8,11,15,16,24-29]$. Table 7 shows the mean width of the maxillary anterior teeth in the ICW found in previous studies $[8,15-17,24,25,29,30]$

\section{Discussion}

This study analyzes the width of the anterior upper teeth in a frontal view and its compliance with the dental esthetic proportions of GP, Preston's
Proportion, RED $70 \%$ and $80 \%$, GPG and MGPG in a Spanish population sample using smile photographs for the first time. The analysis was carried out through a comparison of the mean width ratios of the maxillary anterior teeth and the percentage of compliance of the sample with the values that mark each esthetic proportion, taking into account a deviation of $\pm 1 \%$.

When analyzing the mesiodistal width of the teeth, CE presented slightly higher values on men's teeth $[8.33 \mathrm{~mm}(0.72)$ left and $8.54 \mathrm{~mm}$ (0.71) right] than those registered in Iranian and Thai populations [11,29] and lower than the average recorded in a Turkish [10] sample. On the contrary, the measurements of the lateral incisors and the canines observed by these authors concurred with the ranges described in this study. If the sample is segmented according to sex, both men and women present the mean within the range of values described by other authors in Thai [29], Turkish [27] and Saudi [31] populations.

Forster et al. [12] did not find statistically significant differences when comparing tooth width according to sex, although they did register higher values for men's teeth. This study found significant differences in the left CE $(P=0.028)$ and in the intercanine space $(P=0.005)$, which was also larger in the male sample. Other authors, analyzing each tooth individually, observed that the width of the CE [31], the CA [29] or both [10] teeth in men were significantly larger. On the contrary, Ozdemir et al. show a sample in which the CE width values in women are higher than in men [27].

So far, different works have analyzed the dental esthetic proportions using standardized smile photographs and have compared the values accepted in each norm with the mean values observed in their studies (Fig. 3) (Table 6). The mean width ratios recorded in this study are above the ranges described by previous authors in the right CA/LA ratios of all participants $(86.86 \%$ vs $66.00-85.72 \%)$ [11,15,19,24,26,29] and of men (88.46\% vs. $74.14-86.00 \%)[8,15,16,25,27-29]$. These ratios are below the range in left LA/CE of women (66.13\% vs. $67.00-74.08 \%)$ and right LA/ 
Table 6 Mean values of the tooth width ratios registered in previous studie

\begin{tabular}{|c|c|c|c|c|c|c|c|c|c|c|c|}
\hline Autor and year & Country & Sample & Age range & Left LA / CE (\%) & SD & Right CA / LA (\%) & SD & Left LA / CE (\%) & SD & Left CA / LA $(\%)$ & SD \\
\hline \multicolumn{12}{|l|}{ All participants } \\
\hline Aziz 2017 [19] & India & 100 & $18-35$ & 68.3 & 5.6 & 78.4 & 11.8 & 67.5 & 5.8 & 79.0 & 13.3 \\
\hline Calçada et al. 2014 [24] & Portugal & 50 & n.r & 65.7 & n.r & 85.5 & n.r & 65.1 & n.r & 85.8 & n.r \\
\hline Murthy 2008 [15] & India & 56 & $20-25$ & 70.4 & 6.8 & 82.8 & 10.9 & 69.7 & 7.4 & 80.8 & 9.4 \\
\hline Parnia et al. 2010 [11] & Iran & 100 & $20-27$ & 66.0 & 6.9 & 66.0 & 13 & 63.0 & 8.0 & 68.0 & 13.0 \\
\hline Ramirez et al. 2016 [26] & Colombia & 351 & $18-40$ & 69.0 & 6.0 & 82.0 & 14 & 68.0 & 6.0 & 82.0 & 13.0 \\
\hline Range & & & & $65.7-72.0$ & & $66.0-85.7$ & & $63.0-69.7$ & & $68.0-85.8$ & \\
\hline \multicolumn{12}{|l|}{ Female } \\
\hline Agrawal et al. 2016 [8] & India & 41 & $20-23$ & 74.6 & 9.6 & 84.5 & 16.0 & 74.1 & 8.0 & 82.3 & 14.3 \\
\hline \multirow[t]{2}{*}{ Kantrong et al. 2019 [29] } & Thailand & 85 & n.r & 72.0 & 5.0 & 78.0 & 11.0 & 72.0 & 7.0 & 80.0 & 13.0 \\
\hline & Nepal (A.) & 15 & n.r & 71.2 & 4.2 & 75.8 & 9.2 & 71.9 & 3.8 & 71.2 & 9.9 \\
\hline Maharjan 2018 [16] & Nepal (M.) & 15 & n.r & 68.5 & 10.6 & 77.8 & 16.9 & 70.0 & 10.6 & 71.0 & 13.5 \\
\hline Murthy 2008 [15] & India & 36 & $20-25$ & 70.4 & 6.6 & 82.5 & 11.2 & 69.5 & 6.9 & 79.0 & 9.6 \\
\hline Niranjan et al. 2016 [25] & Saudi Arabia & 30 & $18-30$ & 70.0 & 5.6 & 78.1 & 11.2 & 68.6 & 6.7 & 75.7 & 10.7 \\
\hline Özdemir et al. 2018 [27] & Turkey & 69 & $18-24$ & 67.0 & 5.1 & 88.0 & 10.0 & 67.0 & 5.2 & 88.0 & 9.9 \\
\hline \multicolumn{12}{|l|}{ Male } \\
\hline Agrawal et al. 2016 [8] & India & 39 & $20-23$ & 75.8 & 20.9 & 83.6 & 20.0 & 76.1 & 11.0 & 82.9 & 19.2 \\
\hline \multirow[t]{2}{*}{ Kantrong et al. 2019 [29] } & Thailand & 55 & $\mathrm{n} . \mathrm{r}$ & 72.0 & 6.0 & 82.0 & 14.0 & 73.0 & 6.0 & 83.0 & 12.0 \\
\hline & Nepal (A.) & 18 & n.r & 68.3 & 5.5 & 78.9 & 12.9 & 69.4 & 7.4 & 74.3 & 11.8 \\
\hline Maharjan 2018 [16] & Nepal (M.) & 15 & $\mathrm{n} . \mathrm{r}$ & 69.5 & 5.6 & 79.2 & 8.3 & 69.1 & 8.3 & 73.2 & 12.5 \\
\hline Murthy 2008 [15] & India & 20 & $20-25$ & 70.3 & 6.0 & 83.3 & 10.6 & 69.9 & 8.3 & 82.2 & 9.1 \\
\hline Niranjan et al. 2016 [25] & Saudi Arabia & 30 & $18-30$ & 67.4 & 7.8 & 80.7 & 11.3 & 67.5 & 5.2 & 80.5 & 11.2 \\
\hline Özdemir et al. 2018 [27] & Turkey & 81 & $18-24$ & 69.0 & 6.5 & 86.0 & 1.1 & 69.0 & 6.7 & 87.0 & 10.8 \\
\hline Venkatesh et al. 2018 [28] & India & 51 & $19-23$ & 72.0 & 6.5 & 74.1 & 10.1 & 70.6 & 7.8 & 73.7 & 9.7 \\
\hline Range & & & & $67.4-75.8$ & & $74.1-86.0$ & & $67.5-76.1$ & & $73.2-87.0$ & \\
\hline
\end{tabular}

LA, lateral incisor; CE, central incisor; CA, canine; SD, standard deviation; A., Aryans; M., Mongoloids; n.r, not registered

Table 7 Tooth widths in percentages within the ICW registered in previous studies

\begin{tabular}{|c|c|c|c|c|c|c|c|c|c|c|c|c|c|c|c|}
\hline Author and year & Country & Sample & Age range & Right CA $(\%)$ & SD & Right LA (\%) & SD & Right CE (\%) & SD & Left CE $(\%)$ & SD & Left LA $(\%)$ & SD & Left CA $(\%)$ & SD \\
\hline \multicolumn{16}{|l|}{ All participants } \\
\hline Agrawal et al. 2016 [8] & India & 80 & $20-23$ & 13.0 & n.r & 15.5 & n.r & 21.5 & n.r & 21.5 & n.r & 15.5 & n.r & 13.0 & n.r \\
\hline Aziz 2017 [19] & Portugal & 100 & $18-35$ & 11.9 & 1.3 & 15.2 & 1.1 & 22.6 & 0.9 & 22.8 & 0.9 & 15.5 & 1.0 & 12.1 & 1.2 \\
\hline Kantrong et al. 2019 [29] & Thailand & 140 & n.r & 12.5 & 0.1 & 15.8 & 0.9 & 21.5 & 0.3 & 21.2 & 0.3 & 15.7 & 0.1 & 12.6 & 0.1 \\
\hline Ong et al. $2006[30]$ & UK & 50 & n.r & 13.2 & 2.1 & 14.4 & 1.7 & 23.2 & 1.4 & 23.1 & 1.2 & 13.9 & 1.9 & 12.3 & 1.7 \\
\hline Range & & & & $11.9-13.2$ & & $14.4-15.8$ & & $21.5-23.3$ & & $21.2-23.1$ & & $13.9-15.7$ & & $12.3-13.0$ & \\
\hline \multirow[t]{2}{*}{ Ali Fayaad et al. 2006 [17] } & Jordan & 183 & $20-23$ & 11.9 & 1.6 & 15.2 & 1.3 & 23.0 & 1.1 & 22.8 & 1.3 & 14.6 & 1.41 & 11.7 & 1.7 \\
\hline & Nepal (A.) & 15 & n.r & 12.1 & 1.1 & 15.9 & 0.9 & 22.5 & 1.1 & 22.2 & 0.6 & 15.9 & 0.9 & 11.3 & 17 \\
\hline Maharjan 2018 [16] & Nepal (M.) & 15 & $\mathrm{n} . \mathrm{r}$ & 11.9 & 2.1 & 15.5 & 1.3 & 22.9 & 2.2 & 22.7 & 1.1 & 15.9 & 1.9 & 11.3 & 1.7 \\
\hline Murthy 2008 [15] & India & 36 & $20-25$ & 12.6 & n.r & 15.4 & n.r & 21.9 & n.r & 22.3 & n.r & 15.5 & n.r & 12.3 & n.r \\
\hline Niranjan et al. 2016 [25] & Saudi Arabia & 30 & $18-30$ & 11.8 & 1.6 & 16 & 1.4 & 22.9 & 2.0 & 22.8 & 1.2 & 15 & 1.7 & 11.2 & 1.6 \\
\hline Ong et al. 2006 [30] & UK & 28 & n.r & 12.5 & 2.0 & 14.6 & 1.7 & 23.3 & 1.4 & 23.2 & 1.1 & 14.3 & 1.3 & 12.2 & 1.6 \\
\hline Range & & & & $11.8-12.6$ & & $14.6-16.0$ & & $21.9-23.3$ & & $22.1-23.3$ & & $14.3-16.0$ & & $11.2-12.2$ & \\
\hline \multirow[t]{2}{*}{ Ali Fayaad et al. 2006 [17] } & Jordan & 193 & $20-23$ & 11.7 & 1.6 & 15.1 & 1.0 & 22.7 & 1.3 & 22.6 & 1.3 & 14.6 & 1.1 & 11.9 & 1.4 \\
\hline & Nepal (A.) & 18 & n.r & 12.1 & 1.8 & 15.4 & 0.9 & 22.61 & 1.0 & 22.7 & 1.3 & 15.7 & 1.2 & 11.5 & 1.5 \\
\hline Maharjan 2018 [16] & Nepal (M.) & 15 & n.r & 12.4 & 1.4 & 15.7 & 1.2 & 22.6 & 1.28 & 22.6 & 1.7 & 15.5 & 1.3 & 11.3 & 1.4 \\
\hline Murthy 2008 [15] & India & 20 & $20-25$ & 12.8 & n.r & 15.4 & n.r & 22.0 & $\mathrm{n} . \mathrm{r}$ & 22.0 & n.r & 15.3 & n.r & 12.5 & n.r \\
\hline Niranjan et al. 2016 [25] & Saudi Arabia & 30 & $18-30$ & 12.4 & 1.4 & 15.2 & 0.9 & 22.5 & 1.1 & 22.5 & 0.9 & 15.1 & 1.1 & 12.3 & 1.4 \\
\hline Ong et al. 2006 [30] & UK & 22 & n.r & 14.0 & 1.9 & 14.1 & 1.7 & 23.1 & 1.4 & 23 & 1.2 & 13.5 & 2.4 & 12.4 & 2 \\
\hline Range & & & & $11.7-14.0$ & & $14.1-15.7$ & & $22.0-23.1$ & & $22.0-23.0$ & & $13.5-15.7$ & & $11.3-12.4$ & \\
\hline
\end{tabular}

CA, canine; LA, lateral incisor; CE, central incisor; SD, standard deviation; A., Aryans; M., Mongoloids; UK, United Kingdom; n.r, not registered

CE of men (66.56\% vs. 67.40-75.77\%) [8,15,16,25,27-29].

When comparing the mean tooth width ratios with the GP standard, significant results that confirm compliance with this standard were not found, thus ratifying the findings of Asian [8-11,15,16] and European [12,22,24] studies. Different authors report that GP standards are met in less than $30 \%$ of the cases $[13-17,19,20]$. In other studies, the guidelines are confirmed in a higher number of subjects in the relationship LA/CE than in the relationship CA/LA $[18,22]$. The percentages of GP compliance recorded in this study were less than $5 \%$ except in the left LA/CE ratio, which was less than $17 \%$. These results are similar to those reported by other authors who analyzed samples of individuals from India $(<18 \%$ and $8 \%)[8,19]$ UK (10\% and $1.4 \%$ ) [22] and Nepal (14.20\% and 12.69\%) [16].

In this study, the fulfilment of Preston's proportion was registered in the ratios on the left side of all participants $(P=0.69$ and $P=0.389)$, in all the comparisons of men $(P>0.05)$ and in those of women except between LA/CE right $(P=0.003)$. These data confirm the findings of Calçada et al. in their study on a Portuguese population [24]. However, when analyzing the compliance percentages recorded, they were less than $25 \%$ in $\mathrm{LA} / \mathrm{CE}$ and less than $10.5 \%$ in $\mathrm{CA} / \mathrm{LA}$, which is in line with the observations of Kalia et al. in their UK sample (16\% and 6.55\%) [22]. The present study indicated that the highest percentage of compliance with the Preston norm was registered in the right LA/CE ratio in women $(25 \%)$ and that the lowest compliance percentages were those of men. This may indicate that Preston's proposed values of $66 \%$ in LA/CE and $84 \%$ in CA/LA coincide with the mean width ratios of maxillary anterior teeth observed in WesternEuropean populations. Nevertheless, these conclusions are not applicable 
for clinical use because they are not the most frequently observed values in natural smiles.

The analysis of RED $70 \%$ or $80 \%$ carried out in this study revealed that the standard was not met in the analyzed sample, since the mean values of the LA/CE and CA/LA ratios are not numerically equal, indicating that the exposure of the maxillary anterior teeth in a frontal view does not steadily decrease from the midline to the canine. The highest degree of non-compliance of RED 70\% occurs in the CA/LA comparison. This greater frontal exposure of the canine has also been observed in two Indian populations that registered figures of $75 \%$ (LA/CE) and 83\% (CA/LA) [8] and $69.9 \%(\mathrm{LA} / \mathrm{CE})$ and $81.5 \%(\mathrm{CA} / \mathrm{LA})$ [15]. In contrast, in other Turkish [27] and Nepalese [16] samples, narrower canines were observed.

The GPG and MGPE standards are based on the calculation of the apparent percentages of the six maxillary anterior teeth in the ICW. The values recorded in the present work are within the ranges described in those previous studies (Table 7), except in the left CA of all participants $(12.22 \%$ vs. $12.30-13.00 \%)[8,18,24,29,30]$. The value of the mean width of the right LA in women $(15.03 \%)$ conformed to GPG $(P=0.913)$. When comparing this study with MGPG, the width of the right CE and left CA adjusted to the norm $(P=0.295$ and $P=0.094)$. In women, LA and CE were adjusted on the right side $(P=0.091$ and $P=0.505)$ and on the left the CE and CA $(P=0.094$ and $P=0.620)$. In men, the CE $(P=0.401$ and $P=0.054)$ and the left CA $(P=0.078)$ were adjusted. When comparing these data with the compliance percentages, a correlation between the mean values that conform to the norm and the highest compliance percentages can be seen. This confirms the statement of Kalia et al. [22] that the scattering of the values obtained is lower and closer to the reference values of MGPG. The percentages of compliance with MGPG are appreciably higher than those obtained with GPG and with the other proportions studied, exceeding $50 \%$ in all comparisons and exceeding 68\% in CE. Nevertheless, the values recorded in this study are lower than those described by Kalia et al., where CE and LA exceeded 70\% and CA 60\% [22].

Although this study has a limitation derived from the size and unequal sex distribution of the sample, its clinical-practical interest lies in the fact that it is the first ever study to compare the compliance with MGPG after the proposal of Kalia et al. [22]. Moreover, this study compares the application of the new norm to the classical esthetic proportions, measuring the width of the upper front teeth, and analyzing the results described in the literature. The data of the compliance percentages recorded in this research (Tables 3,5 ), together with the observation of the standard deviations recorded in previous studies (Tables 6,7), show that the distribution of the values obtained by calculating the width of each maxillary anterior tooth in the ICW is less scattered and, therefore, closer to the mean values than using the method of calculating the LA/CE and CA/LA width ratios. Future studies should be carried out to establish the most suitable deviation range for MGPG and to determine if there are factors that influence the application of deviations in greater frequency in any of the three pairs of the maxillary anterior teeth.

In conclusion, the present study allows us to conclude that GP, RED, GPG, and Preston's proportions are not applicable to treatments that seek a smile reproducing natural principles. However, the values designated by MGPG with a deviation of $\pm 1 \%$ are to a great extent applicable for treatments that aim at a natural smile. The analyzed sample presented percentages of compliance with the values marked by MGPG between $50 \%$ and $66.5 \%$, which confirms the initial hypothesis about the tendency of the general population to comply with a dental esthetic proportion but rejects its strict adaptation to any of the esthetic mathematical norms.

\section{Acknowledgments}

We wish to thank Sergio Fernández Ramos and Sergio Arboleya Fernandez for their valuable help in the development and improvement of the photo analysis protocol and in the development of the illustrations. We are also grateful to Tania Iglesias Cabo, technician in the statistical consulting unit of the University of Oviedo, who performed the statistical analysis and Dr. Sonsoles Olay for her assistance in the organization and management of photo sections with the participants.

\section{Conflict of interest}

The authors have no conflict of interest to declare.

\section{References}

1. Lombardi RE (1973) The principles of visual perception and their clinical application to denture esthetics. J Prosthet Dent 29, 358-382.

2. Levin EI (1978) Dental esthetics and the golden proportion. J Prosthet Dent. 40, 244-252.

3. Preston JD (1993) The golden proportion revisited. J Esthet Dent 5, 247-251.

4. Snow SR (1999) Esthetic smile analysis of maxillary anterior tooth width: the golden percentage. J Esthet Dent 11, 177-184.

5. Ward DH (2001) Proportional smile design using the recurring esthetic dental (red) proportion. Dent Clin North Am 45, 143-154.

6. Ward DH (2008) Using the red proportion to engineer the perfect smile. Dent Today 27, $112,114-117$

7. Ward DH (2015) Proportional smile design: using the recurring esthetic dental proportion to correlate the widths and lengths of the maxillary anterior teeth with the size of the face. Dent Clin North Am 59, 623-638.

8. Agrawal V, Kapoor S, Bhesania D, Shah C (2016) Comparative photographic evaluation of various geometric and mathematical proportions of maxillary anterior teeth: a clinical study. Indian J Dent Res 27, 32-36.

9. Chen YH, Cheng YL, Cheng H, Ya H (2020) Comparison of smile esthetics among celebrities, dentists, and dental students in a Han Chinese population. J Prosthet Dent 123, $845-849$

10. Hasanreisoglu U, Berksun S, Aras K, Arslan I (2005) An analysis of maxillary anterior teeth: facial and dental proportions. J Prosthet Dent 94, 530-558.

11. Parnia F, Hafezeqoran A, Mahboub F, Moslehifard E, Koodaryan R, Moteyagheni R et al. (2010) Proportions of maxillary anterior teeth relative to each other and to golden standard in Tabriz dental faculty students. J Dent Res Dent Clin Dent Prospect 4, 83-86.

12. Forster A, Velez R, Antal M, Nagy K (2013) Width ratios in the anterior maxillary region in a Hungarian population: addition to the golden proportion debate. J Prosthet Dent 110, 211-215.

13. de Castro MVM, Santos NC de M, Ricardo LH (2006) Assessment of the "golden proportion" in agreeable smiles. Quintessence Int 37, 597-604.

14. Saha MK, Margie K, Saha SG, Sandeep D, Saxena D, Vijaywargiya N et al. (2017) Perception of acceptable range of smiles by specialists, general dentists and lay persons and evaluation of different aesthetic paradigms. J Clin Diagn Res 11, 25-28.

15. Murthy BVS, Ramani N (2008) Evaluation of natural smile: golden proportion, red or golden percentage. J Conserv Dent 11, 16-21.

16. Maharjan A, Joshi S (2018) Clinical evaluation of maxillary anterior teeth in relation to golden proportion, red proportion and golden percentage. J Nepal Health Res Counc 16, $11-15$.

17. Fayyad MA, Jamani KD, Aqrabawi J (2006) Geometric and mathematical proportions and their relations to maxillary anterior teeth. J Contemp Dent Pract 7, 62-70.

18. Meshramkar R, Patankar A, Lekha K, Nadiger I (2013) A study to evaluate the prevalence of golden proportion and red proportion in aesthetically pleasing smiles. Eur J Prosthodont Restor Dent 21, 29-33.

19. Aziz M, Hossain MZ (2017) Validity of mathematical proportions in maxillary anterior teeth among Bangladeshi population. Trends Orthod 7, 41-48.

20. Nikgoo A, Alavi K, Alavi K, Mirfazaelian A (2009) Assessment of the golden ratio in pleasing smiles. World J Orthod 10, 224-228.

21. Sandeep N, Satwalekar P, Srinivas S, Reddy CS, Reddy G, Reddy BA (2015) An analysis of maxillary anterior teeth dimensions for the existence of golden proportion: clinical study. J Int Oral Health 7, 18-21.

22. Kalia R (2020) An analysis of the aesthetic proportions of anterior maxillary teeth in a UK population. Br Dent J 228, 449-455.

23. Shetty S, Pitti V, Satish Babu C, Kumar GPS, Jnanadev KR (2011) To evaluate the validity of recurring esthetic dental proportion in natural dentition. J Conserv Dent 14, 314-317.

24. Calçada D, Correia A, Araújo F (2014) Anthropometric analysis of anterior maxillary teeth with digital photography - a study in a Portuguese sample. Int J Esthet Dent 9, 370-380.

25. Niranjan NT, Kanaparthy A, Kanaparthy R, Kiran HY (2016) Photographic and manual evaluation of golden percentage and recurrent aesthetic dental proportion in aesthetic smiles. J Evol Med Dent 5, 2267-2270.

26. Ramírez LMG, Ospina JD, Ballesteros LE (2016) Mestizo anterior teeth's proportions. Int J Morphol 34, 223-231.

27. Özdemir H, Köseoğlu M, Bayindir F (2018) An investigation of the esthetic indicators of maxillary anterior teeth in young Turkish people. J Prosthet Dent 120, 583-588.

28. Venkatesh SB, Shetty S (2018) Evaluation of recurring esthetic dental proportion in natural dentition with an esthetic smile. Pak J Med Health Sci 12, 1867-1870.

29. Kantrong N, Traiveat K, Wongkhantee S (2019) Natural upper anterior teeth display an increasing proportion in mesio-distal direction. J Clin Exp Dent 11, 890-897.

30. Ong E, Brown RA, Richmond S (2006) Peer assessment of dental attractiveness. Am J Orthod Dentofac Orthop 130, 163-169.

31. Swelem AA, Al-Rafah EM (2019) Evaluation of "golden proportion" in Saudi individuals with natural smiles. Saudi Dent J 31, 277-283. 